\title{
High perinatal seroprevalence of cytomegalovirus in northern Taiwan
}

\author{
Mei-Huei Chen, ${ }^{1}$ Pau-Chung Chen, ${ }^{5}$ Suh-Fang Jeng, ${ }^{3}$ Chia-Jung Hsieh, ${ }^{5}$ Feng-Chiao Su, ${ }^{5}$ Hua-Fang Liao, ${ }^{3}$ \\ Yi-Ning Su, ${ }^{2,4}$ Shio-Jean Lin $^{6}$ and Wu-Shiun Hsieh ${ }^{1}$ \\ Department of ${ }^{1}$ Pediatrics and ${ }^{2}$ Medical Genetics, National Taiwan University Hospital, ${ }^{3}$ School and Graduate Institute of Physical Therapy, ${ }^{4}$ Graduate Institute \\ of Clinical Medicine, National Taiwan University College of Medicine, ${ }^{5}$ Institute of Occupational Medicine and Industrial Hygiene, National Taiwan University

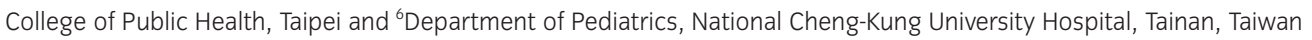

\begin{abstract}
Aim: The living style, health-care system and socio-economic environments have changed substantially in Taiwan over past 20 years. This study was aimed to estimate the current perinatal cytomegalovirus (CMV) seroprevalence in northern Taiwan.

Methods: In a Taiwan Birth Panel Study, 483 pairs of mothers and neonates were prospectively recruited from one tertiary medical center, one local hospital, and two obstetric clinics located in northern Taiwan from April 2004 through January 2005. Sera of their paired maternal and cord blood were tested by an enzyme-linked immunosorbent assay method for CMV IgG and IgM antibodies. Additional data were collected for health measures and epidemiological characteristics through trained interviewers utilising structured questionnaires.

Results: Among 483 mothers studied, 93\% were Taiwanese, 6.4\% were immigrants from the south-eastern Asia and Mainland China, and 0.6\% was aborigines. The seropositive rate of CMV IgG and IgM among the mothers was $91.1 \%$ and $3.5 \%$, respectively. The immigrant mothers and the mothers younger than 20 years of age had a higher IgM seroprevalence $(P<0.05)$. Furthermore, $90.8 \%$ of the offspring had CMV IgG seropositivity and yet none of the neonates were CMV IgM positive.

Conclusion: The seroprevalence of CMV among childbearing women is high in northern Taiwan. The immigrant mothers and the teenage mothers appear to have higher seropositivity of CMV IgM.
\end{abstract}

Key words: cytomegalovirus; perinatal; seroprevalence; Taiwan.

Human cytomegalovirus (CMV) is a human-specific DNA virus that presents in various body fluids and transmits via interpersonal contact. CMV infection often presents with unremarkable symptoms but $1-10 \%$ of infected persons can develop clinical illness. ${ }^{1}$ It is the most common congenital viral infection. Congenital CMV infection may cause the syndrome of cytomegalic inclusion disease as manifested with jaundice, thrombocytopenia, hepatosplenomegaly, petechia, and microcephaly. ${ }^{2}$ Fetus may be infected via both primary and recurrent maternal

\section{Key Points}

1 The perinatal seroprevalence of cytomegalovirus (CMV) is high in northern Taiwan.

2 In Taiwan, there is marked incline in the numbers of adult women emigrated from mainland China, Vietnam, Indonesia, Cambodia and Thailand mainly for marriage.

3 The immigrant mothers and the teenage mothers have higher seropositivity of CMV IgM.

Correspondence: Dr Wu-Shiun Hsieh, Department of Pediatrics, National Taiwan University Hospital and National Taiwan University College of Medicine, No. 7, Chung-Shan South Road, Taipei, Taiwan. Fax: +886 2 23934749; email: hsiehws@ntuh.gov.tw

Accepted for publication 3 July 2007 infection. The vertical transmission rate of the former is about $40-50 \%$, and of the later $0.5-2 \% .^{1,3}$ In addition, perinatal or postnatal CMV infection can occur as a consequence of exposure to genital secretions and breast milk. ${ }^{3,4}$ Although only 5$10 \%$ of infected infants manifest clinical symptoms, most of them may suffer from long-term neurological sequelae. Furthermore, $7-15 \%$ of asymptomatic infants develop hearing impairment in childhood. ${ }^{1,2}$

The incidence of perinatal CMV infection is highly related to maternal seroprevalence rate, which is further affected by socioeconomic status, age, parity, contact of young children and sexual behaviour. ${ }^{1,4-6}$ The CMV seropositivity in female adults has varied widely from $40 \%$ to $80 \%$ in the United States and Western Europe, and up to $90 \%$ to $100 \%$ in southern America, Africa and Asia. ${ }^{1,7-16}$ Twenty-five years ago in Taiwan, the seroprevalence rates of $\mathrm{CMV}$, as determined by a complementfixation method, were $78 \%, 82.5-87.7 \%$ and $95 \%$ among infants, toddlers and female adults, respectively. ${ }^{17}$ During recent decades, the living conditions, sexual behaviour, child rearing practices and public health system have changed in Taiwan. In addition, there is marked increase in the numbers of adult women emigrated from mainland China, Vietnam, Indonesia, Cambodia and Thailand mainly for marriage. The numbers of neonates born to these immigrant mothers is also increasing. ${ }^{18}$ The seropositivity rate of CMV in Finland and Japan were decreasing during recent decades. In Taiwan, the living 
environment is still crowded and yet there are improvements in the healthy care system and socio-economic status. The seropositivity rate might be expected to be lower compared with the data of years ago. The objective of this study was to determine the current perinatal CMV seroprevalence in northern Taiwan.

\section{Methods}

We prospectively recruited consecutive samples of mothers and their neonates from one tertiary medical center, one local hospital, and two obstetric clinics located in northern Taiwan from April 2004 through January 2005. This is a pilot investigation that concerned congenital CMV infection in northern Taiwan for the project of the Taiwan Birth Panel Study. The Institutional Review Board of National Taiwan University Hospital approved the study, and informed consent was obtained from the parents before enrollment of the study. Blood samples obtained from the paired maternal and cord bloods were tested by the enzymelinked immunosorbent assay method for CMV IgG (Immulite, Los Angeles, USA) and IgM antibodies (Euroimmun, Lubeck, Germany). Gestational age, birthweight, and head circumference were measured by well-trained nurses after delivery. Additional epidemiological characteristics and health measures including nationality, maternal age, education, parity, gender, etc. were collected through trained interviewers utilising structured questionnaires.

The maternal and perinatal data including the CMV seroprevalence were analysed. Chi-squared test or Fisher's exact test was used for categorical variables and $t$-test was used for continuous variables. A $P$-value of $<0.05$ was considered significant. The SPSS 11 software for windows was used for statistical analyses.

\section{Results}

In total, 483 mother-neonate pairs were included for analysis. Among the studied mothers, 93\% were Taiwanese, $6.4 \%$ were immigrants from the south-eastern Asia and Mainland China, and $0.6 \%$ was aboriginals. Approximately half of the mothers had educational level of high school or below. In total, $2 \%$ of the mothers were aged below 20 years old. The male to female ratio among the studied neonates was 50.8:49.2. In total, $56.3 \%$ of them were primipara. Furthermore, $8.5 \%$ were preterm birth and $5.8 \%$ had birthweight less than $2500 \mathrm{~g}$ (Table 1). A total of 440 mothers $(91.1 \%, 95 \%$ CI $88.6 \%$ to $93.6 \%$ ) had CMV IgG antibody and $17(3.5 \%, 95 \%$ CI $1.9 \%$ to $5.2 \%)$ were found CMV IgM positive. All the offspring except one among the mothers with CMV IgG positive had CMV IgG seropositivity. None of the neonates were CMV IgM positive, however (Table 2). Detailed characteristics were analysed according to the status of maternal seroprevalence of CMV IgG and IgM (Table 1). The seropositivity of IgM was significantly higher among immigrant mothers compare to Taiwanese mothers $(14.8 \%$ vs. $2.9 \%, P<0.05)$. Maternal age of younger than 20 years old was also identified as a risk factor of having positive CMV IgM titers compare to those of the mothers with age of $\geq 20$ years $(25 \%$ vs. $3.3 \%, P<0.05)$. Although not statistically

Table 1 Demographic and perinatal characteristics of the studied mothers and their offspring

\begin{tabular}{|c|c|c|c|c|c|}
\hline \multirow[t]{2}{*}{ Characteristics } & \multirow[t]{2}{*}{ Total } & \multicolumn{2}{|l|}{ CMV IgG } & \multicolumn{2}{|l|}{ CMV IgM } \\
\hline & & Positive & Negative & Positive & Negative \\
\hline Nationality & 483 & & & & \\
\hline Taiwanese & 452 & 411 & 41 & 13 & 439 \\
\hline Foreign-born & 31 & 29 & 2 & 4 & 27 \\
\hline Age & 483 & & & & \\
\hline$<20$ & 10 & 8 & 2 & 2 & 8 \\
\hline$\geq 20$ & 473 & 432 & 41 & 15 & 458 \\
\hline Education & 483 & & & & \\
\hline High school and below & 235 & 218 & 17 & 10 & 225 \\
\hline College and above & 248 & 222 & 26 & 7 & 241 \\
\hline Parity & $482 \dagger$ & & & & \\
\hline 1 & 229 & 205 & 24 & 10 & 219 \\
\hline 2 & 180 & 163 & 17 & 4 & 176 \\
\hline$\geq 3$ & 73 & 71 & 2 & 3 & 70 \\
\hline Preterm & 483 & & & & \\
\hline No & 442 & 402 & 40 & 15 & 427 \\
\hline Yes & 41 & 38 & 3 & 2 & 39 \\
\hline Infant gender & 483 & & & & \\
\hline Male & 245 & 221 & 24 & 11 & 234 \\
\hline Female & 238 & 219 & 19 & 6 & 232 \\
\hline Mean gestational age & & 38.5 & 38.9 & 37.9 & 38.5 \\
\hline in weeks (SD) & & (1.7) & (1.4) & (1.5) & (1.7) \\
\hline Mean birthweight in & & 3148 & 3159 & 2865 & 3159 \\
\hline grams (SD) & & (492) & (619) & (352) & (506) \\
\hline
\end{tabular}


significant, two features were observed. First, nulliparous mothers had higher incidence of positive IgM titers. Second, the neonates born to IgM seropositivity mothers had lower birthweight.

\section{Discussion}

Since the introduction of widespread rubella immunisation programs, CMV is now the most common viral cause of congenital and perinatal infection. There was no available vaccine for prevention of CMV infection. Currently, three antiviral drugs are licensed for treatment of CMV infection including ganciclovir (and its prodrug, valganciclovir), foscarnet and cidofovir. However, the experience of treatment in congenital and perinatal CMV infection is still limited. ${ }^{19,20}$ Therefore, to identify the risk factors of vertical and perinatal infection, and to decrease the maternal seroprevalence is important. The risk factors documented in literatures include non-white races, lower socioeconomic status, maternal age $<20$ years old, non-married, multigravidity, less than 16 years of education, being breastfed as an infant, the presence of children five to 18 years of age in the home, etc. ${ }^{1,4,5}$

Maternal seroprevalence of CMV are similar in Australia, Europe and North America, varying from $40 \%$ to $60 \%$ in

Table 2 Seroprevalence of CMV IgG and IgM among the studied mothers and their neonates

\begin{tabular}{cclc}
\hline Mothers & & Neonates & Total \\
\hline $\operatorname{lgG}(+) \dagger$ & $\operatorname{lgG}(-)$ & & \\
\hline 439 & 0 & IgG $(+)$ & 439 \\
1 & 43 & IgG $(-)$ & 44 \\
440 & 43 & Subtotal & 483 \\
$\operatorname{lgM}(+)$ & $\operatorname{lgM}(-)$ & & Total \\
0 & 0 & IgM (+) & 0 \\
17 & 466 & IgM (-) & 483 \\
17 & 466 & Subtotal & 483 \\
\hline
\end{tabular}

†lg, immunoglobulin. CMV, cytomegalovirus. population of middle and high socio-economic status to $80 \%$ in those of low socio-economic status. However, the prevalence of a very high rate of pre-existing maternal immunity is noted in Africa and Asia. ${ }^{1}$ During the past two decades, the seropositive rates of CMV have remained unchanged in United States, ${ }^{7,8}$ England $^{9,10}$ and Australia. ${ }^{11,12}$ Whereas, the seroprevalence of CMV in Finland seems to decline since the 1980s (from $84 \%$ to $70.7 \%$ ), ${ }^{13,14}$ and so does in Japan (from 94\% to $\left.68.1 \%\right)^{15,16}$ (Table 3). The decrease in the seroprevalence of CMV may relate to improvement in socio-economic status, hygienic measures and public health-care system. ${ }^{14,16}$ Despite of recent advancement in socio-economic status and public health-care system in Taiwan, our study had demonstrated that there has no substantial change in the seroprevalence of CMV among childbearing women during recent two decades. The reasons for such a persistent high servoprevalence remain unclear. It may be attributed to the lifestyle including the crowded condition, childrearing practice, or hygienic measures.

In this study, we found that the seropositivity of CMV IgM was significantly higher among the immigrant mothers and the mothers with age of less than 20 years. In Taiwan, a unique marriage-based immigration emerged during recent years. The percentage of immigrant mothers increased from 15.7\% in 1998 to $32.1 \%$ in 2003 , and the percentage of neonates born to these mothers increased substantially from $5.1 \%$ in 1998 to $13.8 \%$ in 2004. ${ }^{18,21}$ Moreover, the majority of the immigrant mothers were from the south-eastern Asia and Mainland China. Whether the lifestyle, socio-economic status, or other factors in immigrant mothers influence the seroprevalence of CMV in Taiwan require further investigation.

Several limitations in study design merit consideration. First, our study only examined the CMV IgG and IgM without the determination of avidity value, utility of polymerase chain reaction (PCR) based methods or CMV culture that failed to determine the prevalence of primary or recurrent infection in the mothers or congenital infection in the neonates. Second, the study size of immigrants and the teenage mothers was too small, and further collection of samples is warranted to make a conceded conclusion. Third, this study was performed in northern Taiwan, a geographically distinction that may not be generalised to represent the whole population in Taiwan. Finally, the cited study results involving different countries also had variable

Table 3 Reported seroprevalences of CMV antibodies between 1977 and 2005

\begin{tabular}{|c|c|c|c|c|c|c|c|c|}
\hline \multirow{2}{*}{$\begin{array}{l}\text { Period } \\
\text { Area }\end{array}$} & \multicolumn{4}{|c|}{ 1977-1985 } & \multicolumn{4}{|c|}{ 1991-2004 } \\
\hline & Year & Number & SES & CMV IgG (+) & Year & Number & SES & CMV IgG (+) \\
\hline \multirow[t]{2}{*}{ United States } & \multirow[t]{2}{*}{$1980^{7}$} & 461 & Upper & $50 \%$ & \multirow[t]{2}{*}{$2000^{8}$} & \multirow[t]{2}{*}{2992} & Upper & $40 \%$ \\
\hline & & 493 & Lower & $83 \%$ & & & Lower & $89 \%$ \\
\hline England & $1984^{9}$ & 10847 & NA & $58 \%$ & $1991^{10}$ & 2737 & NA & $60 \%$ \\
\hline Australia & $1985^{11}$ & 47320 & NA & $58-71 \%$ & $2005^{12}$ & 600 & NA & $56.8 \%$ \\
\hline \multirow[t]{2}{*}{ Finland } & \multirow[t]{2}{*}{$1977^{13}$} & \multirow[t]{2}{*}{148} & \multirow[t]{2}{*}{ NA } & \multirow[t]{2}{*}{$84 \%$} & \multirow[t]{2}{*}{$1994^{14}$} & \multirow[t]{2}{*}{1088} & Upper & $60.9 \%$ \\
\hline & & & & & & & Lower & $76.4 \%$ \\
\hline Japan & $1983^{15}$ & 2070 & NA & $94 \%$ & $2000^{16}$ & 345 & NA & $68.1 \%$ \\
\hline Taiwan & $1980^{17}$ & 171 & NA & $95 \%$ & $2004 \dagger$ & 483 & NA & $91.1 \%$ \\
\hline
\end{tabular}

†Current study; NA, information not available. CMV, cytomegalovirus. 
population and study period and may not be adequate for chronological and geographical comparison.

In conclusion, this study demonstrates that a high servoprevalence of CMV is an important public health issue in Taiwan. Our findings have highlighted the emerging importance of CMV as an important perinatal infection among immigrant mothers and teenage mothers in Taiwan. A continued follow up of these infants' health outcomes is undertaken to determine the significance of seroprevalence of CMV.

\section{Acknowledgements}

This study was supported by the grants BHP-PHRC-92-4 and DOH93-HP-1702 awarded by the Bureau of Health Promotion, Department of Health, Taiwan, Republic of China.

\section{References}

1 Gaytant MA, Steegers EA, Semmekrot BA, Merkus HM, Galama JM. Congenital cytomegalovirus infection: review of the epidemiology and outcome. Obstet. Gynecol. Surv. 2002; 57: 245-56.

2 Munro SC, Trinocado D, Hall B, Rawlinson WD. Symptomatic infant characteristics of congenital cytomegalovirus disease in Australia. J. Paediatr. Child. Health 2005; 41: 449-52.

3 Stagno S, Pass RF, Dworsky ME, Alford CA. Maternal cytomegalovirus infection and perinatal transmission. Clin. Obstet. Gynecol. 1982; 25: 563-76.

4 Murph JR, Souza IE, Dawson JD et al. Epidemiology of congenital cytomegalovirus infection: maternal risk factors and molecular analysis of cytomegalovirus strains. Am. J. Epidemiol. 1998; 147: 940-7.

5 Fowler KB, Stagno S, Pass RF. Maternal age and congenital cytomegalovirus infection: screening of two diverse newborn populations, 1980-1990. J. Infect. Dis. 1993; 168: 552-6.

6 Jones CA. Neonatology for the generalist: maternal transmission of infectious pathogens in breast milk. J. Paediatr. Child. Health 2001; 37: 576-82.

7 Montgomery JR, Mason EO, Williamson AP, Desmond MM, South MA. Prospective study of congenital cytomegalovirus infection. South Med. J. 1980; 73: 590-5.
8 Marshall GS, Stout GG. Cytomegalovirus seroprevalence among women of childbearing age during a 10-year period. Am. J. Perinatol. 2005; 22: 371-6.

9 Griffiths PD, Baboonian C. A prospective study of primary cytomegalovirus infection during pregnancy: final report. Br. J. Obstet. Gynaecol. 1984; 91: 307-15.

10 Griffiths PD, Baboonian C, Rutter D, Peckham C. Congenital and maternal cytomegalovirus infections in a London population. Br. J. Obstet. Gynaecol. 1991; 98: 135-40.

11 Hatherley LI. The incidence of cytomegalic inclusion disease (CID) in an obstetric teaching hospital 1975-1984. Aust. NZ. J. Obstet. Gynaecol. 1985; 25: 171-5.

12 Munro SC, Hall B, Whybin LR et al. Diagnosis of and screening for cytomegalovirus infection in pregnant women. J. Clin. Microbiol. 2005; 43: 4713-18.

13 Granstrom M, Leinikki P, Santavuori P, Pettay O. Perinatal cytomegalovirus infection in Man. Arch. Dis. Child. 1977; 52: 354-9.

14 Mustakangas P, Sarna S, Ammala P, Muttilainen M, Koskela P, Koskiniemi M. Human cytomegalovirus seroprevalence in three socioeconomically different urban areas during the first trimester: a population-based cohort study. Int. J. Epidemiol. 2000; 29: 587-91.

15 Kamada M, Komori A, Chiba S, Nakao T. A prospective study of congenital cytomegalovirus infection in Japan. Scand. J. Infect. Dis. 1983; 15: 227-32.

16 Numazaki K, Fujikawa T. Prevalence of serum antibodies to cytomegalovirus in pregnant women in Sapporo, Japan. Int. J. Infect. Dis. 2002; 6: 147-8.

17 Wang TR. Seroepidemiological survey of cytomegalovirus infections in the urban and suburban areas of Taipei city. J. Formos. Med. Assoc. 1980; 79: 753-9.

18 Hsieh WS, Wu HC, Jeng SF et al. Nationwide singleton birth weight percentiles by gestational age in Taiwan, 1998-2002. Acta. Paediatr. Taiwan 2006; 47: 25-33.

19 Schleiss MR. Antiviral therapy of congenital cytomegalovirus infection. Semin. Pediatr. Infect. Dis. 2005; 16: 50-9.

20 Vancikova Z, Kucerova T, Pelikan L, Zikmundova L, Prigolva M. Perinatal cytomegalovirus hepatitis: to treat or not treat with ganciclovir. J. Paediatr. Child. Health 2004; 40: 444-8.

21 Ministry of the Interior, R.O.C. Marriage and family. Available from: http://www.moi.gov.tw/stat/gender/ps03-04.xls [accessed 10 January 2006]. 